EDITORIAL

\title{
NPP (Neuropsychopharmacology): update on gender balance in journal function
}

Neuropsychopharmacology (2019) 44:2145-2148; https://doi.org/ 10.1038/s41386-019-0513-6

Last year (2018) we provided an initial report on gender balance in NPP (Neuropsychopharmacology) function [1]. Specifically, we examined the representation of men and women in editorial,

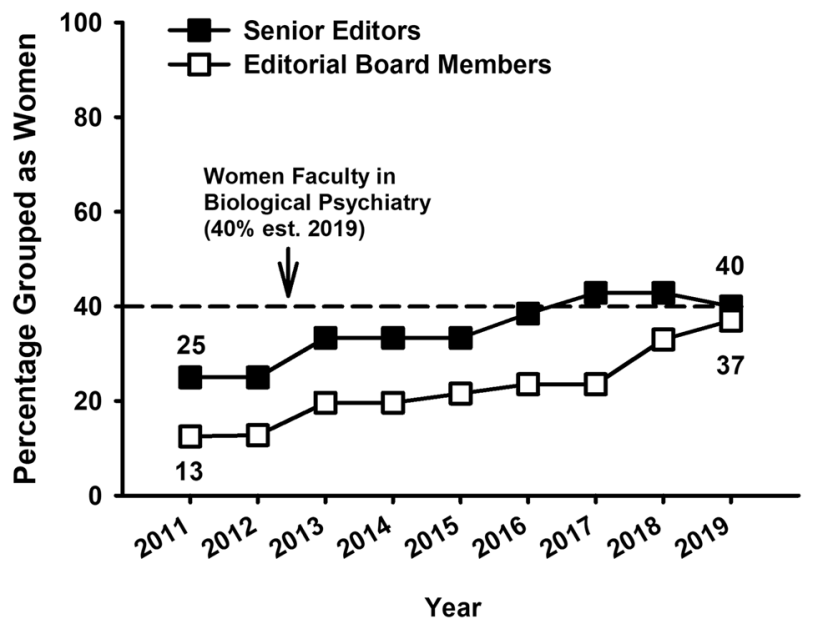

Fig. 1 Editorial roles in NPP from 2011 through 2019. The year 2011 is the first year for which detailed data sets became available. Percentage of women serving as Senior Editors (filled squares) and on the Editorial Board (open squares) by year

\section{A. Invited Reviewers}

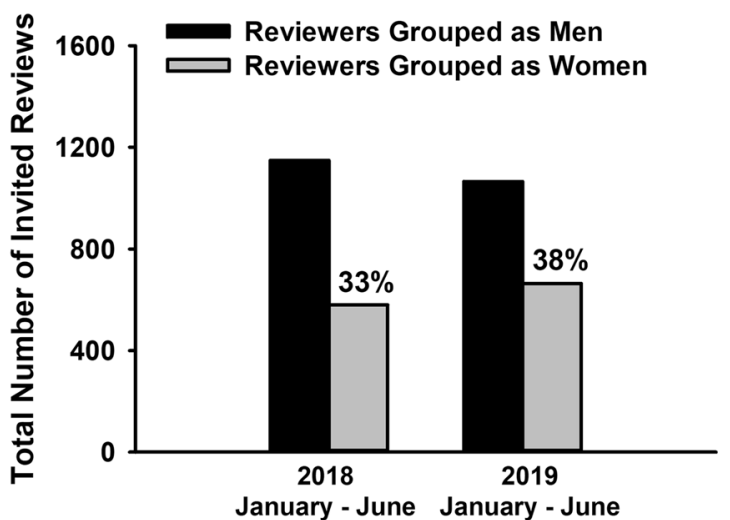

reviewer, and authorship roles at NPP. Our findings indicated that, in general, gender balance across these roles was encouraging and had been improving over time. We also identified restricted areas that would benefit from improved gender balance. Here we provide an update on the actions we took and how these metrics have changed over the last year.

In our initial report [1], we used https://biaswatchneuro.com/ to estimate gender balance across our field. We used their estimate for faculty-level ranks, since in general it is faculty members that serve in the roles that we studied for this special project (i.e., Senior Editors, Editorial Board Members, Reviewers, and Corresponding authors). We acknowledge that by restricting our estimates to these roles, we may be missing early-career researchers who have engaged in roles that (at least currently) are not typical for trainees. We originally used the estimate of the proportion of women faculty engaged in neuroscience research (39\%) as a proxy for the composition of the ACNP (American College of Neuropsychopharmacology) - the society represented by NPP. For this update, new data are available for the field of "Biological Psychiatry," which is a better fit with the full scope of activities of the ACNP membership. Accordingly, here we have adjusted our estimate of women faculty slightly upward, to $40 \%$. We did not perform statistical analyses on differences between 2018 and 2019 because at this time we are primarily interested in whether changes are trending toward improvement or deterioration.

Updated analysis of Editorial roles indicates that the proportions are at, or very near, the $40 \%$ estimate of the proportion of women faculty in the field (Fig. 1). In particular, for the editorial board-considered to be the journal's most active and loyal

\section{B. Completed Reviews}

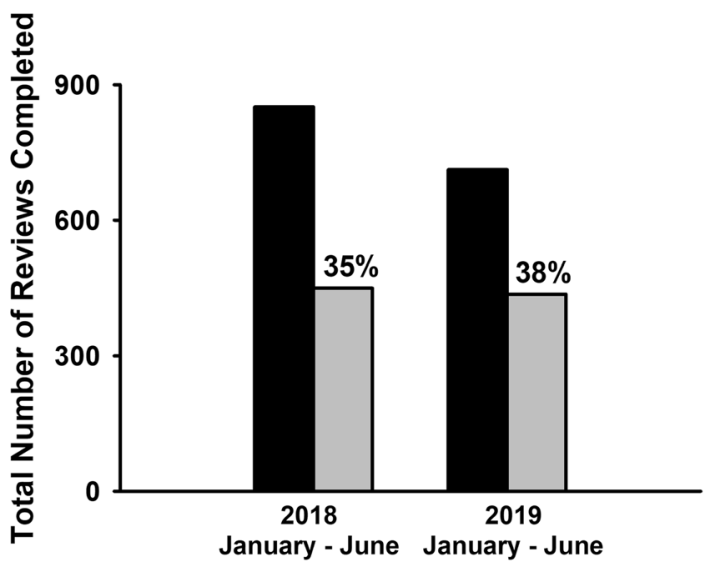

Fig. 2 Reviewers for NPP during a 6-month period in 2018 (pre-report; January-June) and the same period in 2019 (post-report; January-June). a Total number of reviewers grouped as men (black bars) or women (gray bars). b Total number of reviews completed by reviewers grouped as men or women 


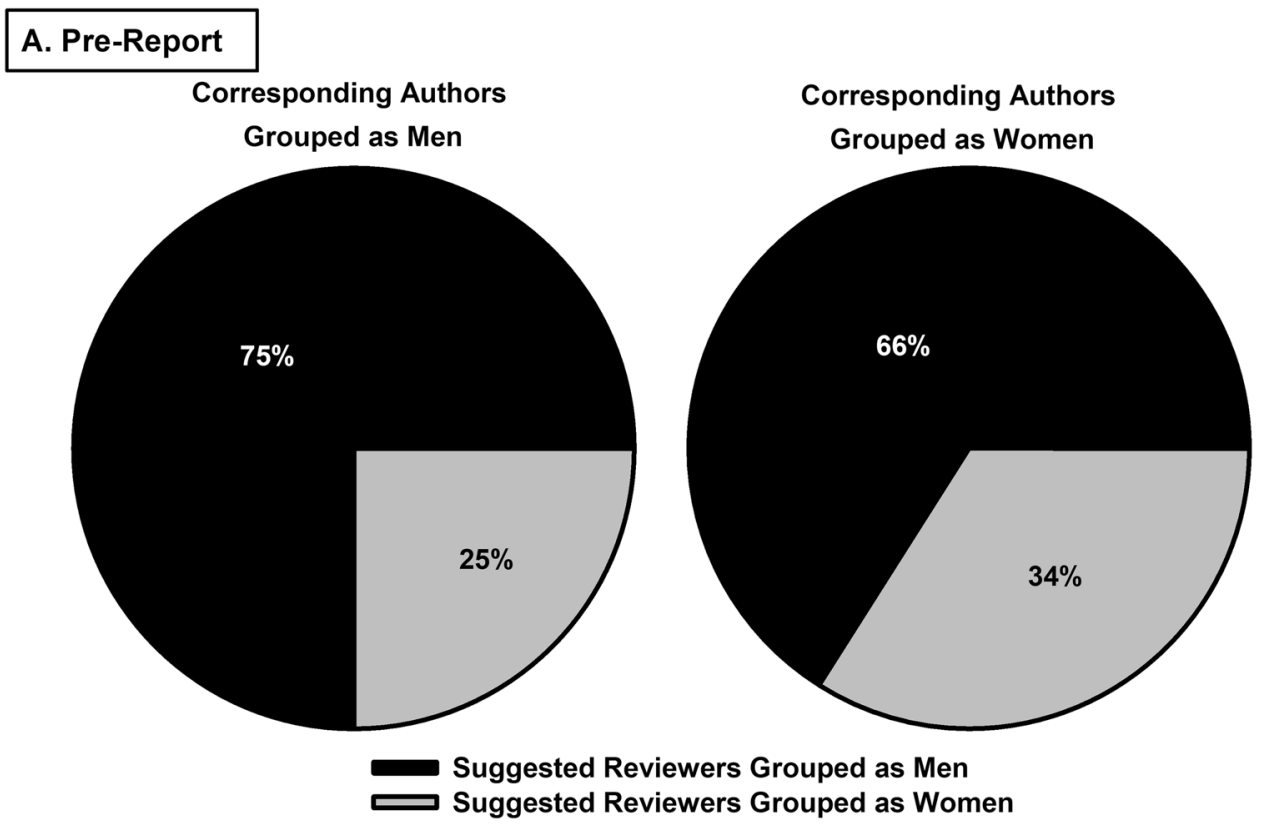

$N=583$ manuscripts
January - June 2018

\section{B. Post-Report}
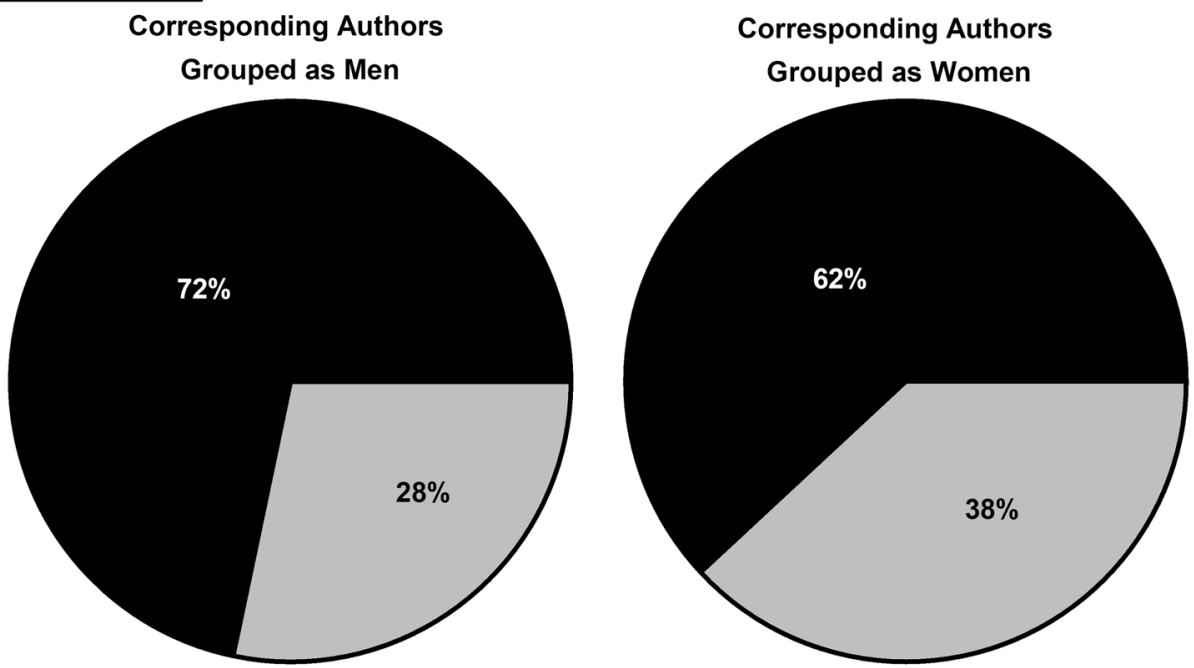

Suggested Reviewers Grouped as Men

Suggested Reviewers Grouped as Women

\section{$\mathrm{N}=608$ manuscripts \\ January - June 2019}

Fig. 3 Reviewers suggested to include by corresponding author during a 6-month period in 2018 (pre-report; January-June) and the same period in 2019 (post-report; January-June). a Pre-report; left: percentage of suggested reviewers grouped as men (black segment) and women (gray segment) among corresponding authors grouped as men. Right: percentage of suggested reviewers grouped as men and women among corresponding authors grouped as women. b Post-report; left: percentage of suggested reviewers grouped as men (black segment) and women (gray segment) among corresponding authors grouped as men. Right: percentage of suggested reviewers grouped as men and women among corresponding authors grouped as women

reviewers - there has been a consistent increase in the proportion of women members that began with the transition to the current journal leadership (in 2013). The current trajectory should enable NPP to exceed estimates of women in our field within the next 1-2 years.

For reviewer roles, we originally collected data in 2018 over a 6month period (January-June). Updated figures for 2019 cover the exact same 6-month period (January-June), acknowledging that submission patterns often differ throughout the year. Gender balance in invitations to review (Fig. 2a) and the number of reviews completed (Fig. 2b) continue to improve and currently approach the $40 \%$ estimate for the proportion of women faculty in fields related to the subject matter covered in NPP.

As described in our initial report [1], membership on NPP's Editorial Board has been based on objective metrics of performance 


\section{A. Pre-Report}

Corresponding Authors

Grouped as Men

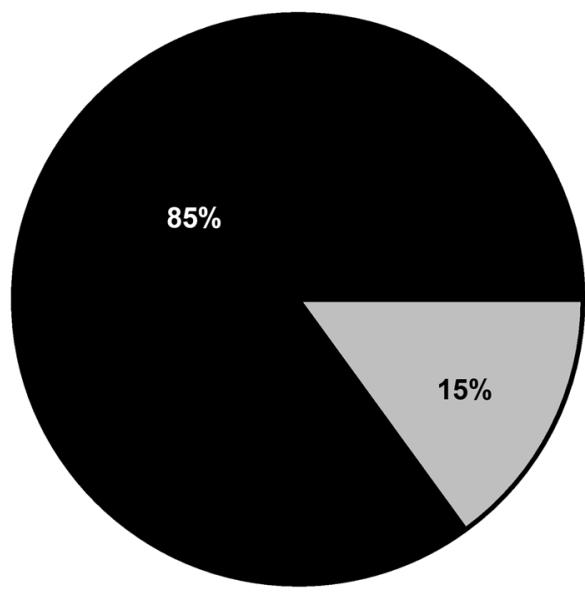

Excluded Reviewers Grouped as Men

Excluded Reviewers Grouped as Women

$\mathrm{N}=\mathbf{5 8 3}$ manuscripts

January - June 2018

\section{B. Post-Report}

Corresponding Authors

Grouped as Men

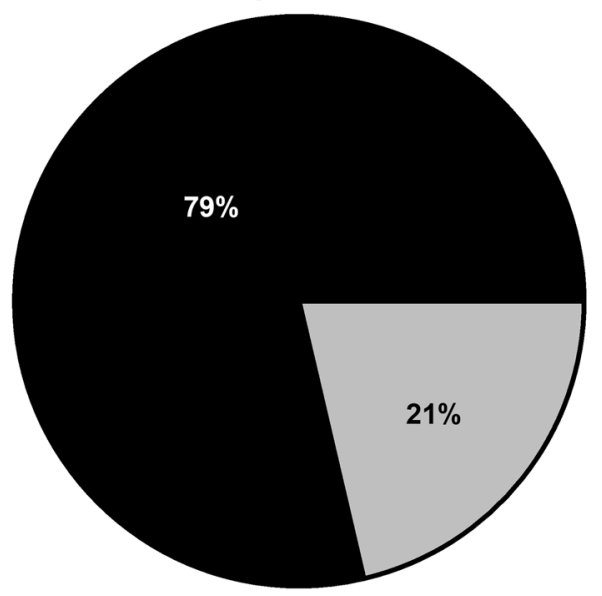

Corresponding Authors

Grouped as Women

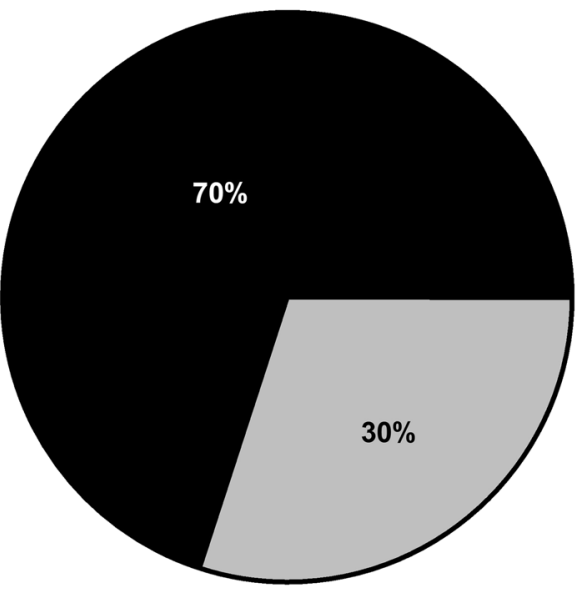

$30 \%$

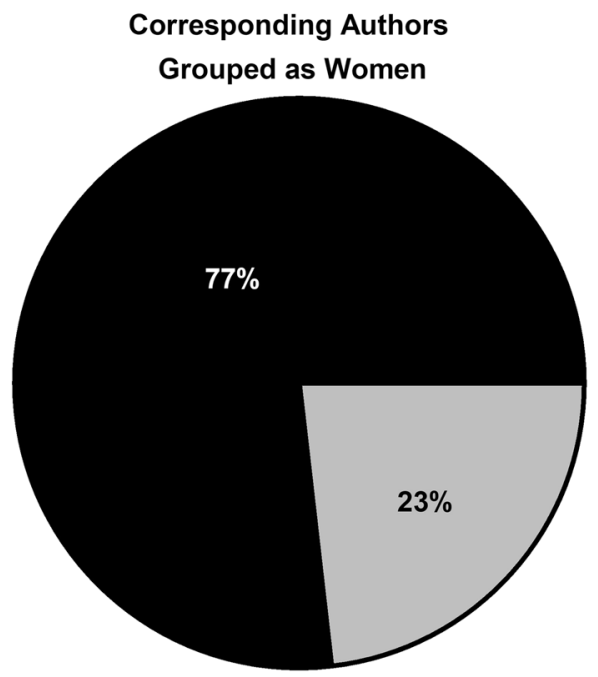

Corresponding Authors

Excluded Reviewers Grouped as Women

\section{$\mathrm{N}=608$ manuscripts \\ January - June 2019}

Fig. 4 Reviewers suggested to exclude by corresponding author during a 6-month period in 2018 (pre-report; January-June) and the same period in 2019 (post-report; January-June). a Pre-report; left: percentage of excluded reviewers grouped as men (black segment) and women (gray segment) among corresponding authors grouped as men. Right: percentage of excluded reviewers grouped as men and women among corresponding authors grouped as women. b Post-report; left: percentage of excluded reviewers grouped as men (black segment) and women (gray segment) among corresponding authors grouped as men. Right: percentage of excluded reviewers grouped as men and women among corresponding authors grouped as women

as a reviewer. Because NPP Senior Editors are empowered to use author input for suggested and excluded reviewers, we explored the possibility that women are less frequently suggested as reviewers during the online submission process. If so, this would create a situation where the Editorial goal of respecting author suggestions for qualified reviewers has the unintended consequence of fewer women being included in the reviewer pool and ultimately being promoted to the Editorial Board on the basis of performance metrics. It is important to note that on the basis of the instructions provided to authors during the online submission process, either gender could hypothetically suggest $100 \%$ of their own gender as qualified reviewers. Despite the latitude offered in the process, we discovered that both men and women were far more likely to suggest men as reviewers (Fig. 3a). 
A. Corresponding Authors of Submissions

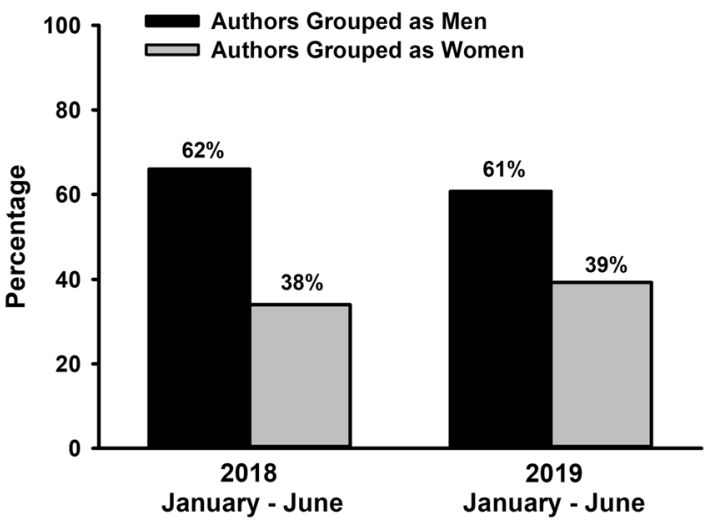

B. Corresponding Authors of Acceptances

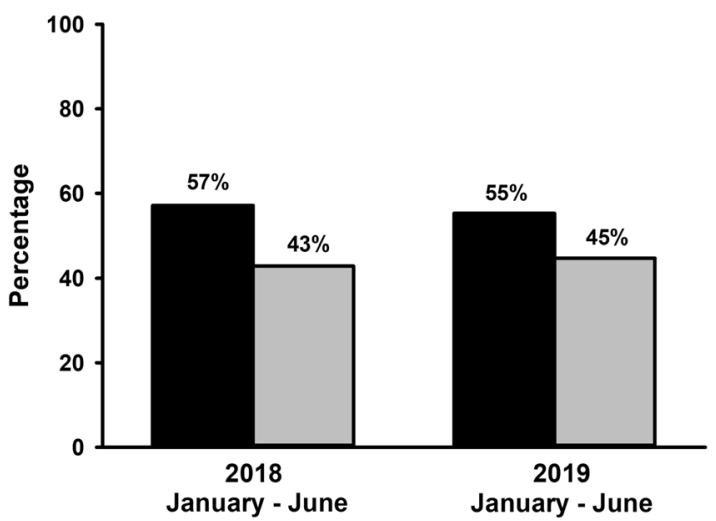

Fig. 5 Corresponding authors of submitted and accepted manuscripts during a 6-month period in 2018 (pre-report; January-June) and the same period in 2019 (post-report; January-June). a Percentage of men (black bars) and women (gray bars) submitting to NPP as corresponding authors. b Percentage of men (black bars) and women (gray bars) who were corresponding authors of accepted manuscripts in NPP

In response to this discovery, we instituted a program by which Senior (Handling) Editors were encouraged to be more mindful of gender balance of their reviewer pool, without mandating strict balance in reviewer proportions. We elected to not mandate a strict balance because, depending upon reviewer availability, this would dramatically increase the time to complete reviews, which NPP author surveys have revealed as a top priority for the authorship pool. Moreover, we were hesitant to impose any measures that would over-burden ("tax") individuals represented in the smaller group. Also, on July 1, 2018 we added a statement to the instructions to authors stating the journal's goal to promote diversity across all journal functions and encouraging author participation in this process when constructing their list of suggested reviewers. The specific statements are: "Please list the names of 6 experts who are knowledgeable in your area and could give an unbiased review of your work", and "NPP Editors wish to enhance diversity in all journal functions, including the composition of our reviewer pool, and emphasize that this is an opportunity for authors to participate in this process".

Following the release of our report and implementation of these interventions, in 2019 we saw a nominal improvement in the gender distribution of reviewers suggested by both men and women (Fig. 3b), although both genders continue to suggest a higher proportion of men than women. Analysis of the gender distributions of excluded reviewers (Fig. 4a, b) indicated that while men are excluding progressively more women as reviewers postintervention, women are excluding fewer women as reviewers. While the meaning of these changes is difficult to ascertain without more information, a sanguine interpretation that can be applied to both trends is that both men and women authors are increasingly envisioning women as qualified and fair reviewers for their manuscripts.

Finally, analysis of the gender of corresponding authors on submitted manuscripts (Fig. 5a) and accepted papers (Fig. 5b) indicate incremental improvements over the last year, while already meeting or exceeding the estimates of women faculty in our field.

We acknowledge that the levels of analyses used for this special project do not enable us to determine whether NPP efforts to increase mindfulness of gender balance in journal function played a role in improving these metrics over the past year, or if the improvements reflect a gradual shift in norms that would have occurred regardless. A recent report indicates general trends towards improved gender balance in authorship at leading psychiatry journals [2]. We continue to be mindful that exceeding the actual proportion of women in our field could represent a path toward creating excessive burdens. Indeed, an informal sampling of social media conversations in response to our initial report revealed significant concerns about processes that can create over-burdening, which tends to produce outcomes (e.g., poor reviewer metrics) that are incongruent with the intended goal of increasing representation. NPP leadership will continue to engage in efforts that are intended to ensure that opportunities as editors, reviewers, and authors align with the demographics of the ACNP and associated fields of research, while promoting inclusion and demographic diversity at all levels of journal function.

\section{FUNDING AND DISCLOSURE}

The authors have formal roles at NPP: CJJ is an Editorial Intern and WAC is the Editor-in-Chief.

\section{ACKNOWLEDGEMENTS}

CJJ completed this project as part of an NPP Editorial Internship. We thank staff in the NPP editorial office (Terri Bowen, Lori Kunath, Jennifer Mahar) for providing data sets in formats that would not reveal privileged information, and Dr. Carolyn Rodriguez and Dr. Tony George for providing comments on early versions of the paper.

\section{ADDITIONAL INFORMATION}

Publisher's note Springer Nature remains neutral with regard to jurisdictional claims in published maps and institutional affiliations.

Chloe J. Jordan (D) and William A. Carlezon Jr. (D) ${ }^{2}$ ${ }^{1}$ Molecular Targets and Medications Discovery Branch, National Institute on Drug Abuse, Intramural Research Program, Baltimore, MD 21224, USA and ${ }^{2}$ Department of Psychiatry, McLean Hospital, Harvard Medical School, Belmont, MA 02478, USA Correspondence: Chloe J. Jordan (chloe.jordan@nih.gov)

\section{REFERENCES}

1. Jordan CJ, Carlezon WA Jr. Neuropsychopharmacology (NPP): gender balance in journal function. Neuropsychopharmacology. 2019;44:4-8.

2. Hart KL, Frangou S, Perlis RH. Gender trends in authorship in psychiatry journals from 2008 to 2018. Biol Psychiatry. 2019. In press. Available online 19 February 2019. 